\title{
Identification of Aqueous Reservoirs in Poly(alkylcyanoacrylate) Nanoparticles
}

O. Thomas ${ }^{\mathrm{a}}$, L. Lemaire ${ }^{\mathrm{a}}$, J.P. Benoit ${ }^{\mathrm{a}}$ and P. Saulnier ${ }^{\mathrm{a}}$

${ }^{a}$ Micro et Nanomedecines translationnelles, MINT, UNIV Angers, INSERM 1066, CNRS 6021, 4 rue Larrey, Angers, France

Paper published in Colloid and Polymer Science

Volume 298, January 2020, Pages 1-10

https://doi.org/10.1007/s00396-019-04593-4

Correspondence to: olivier.thomas@univ-angers.fr

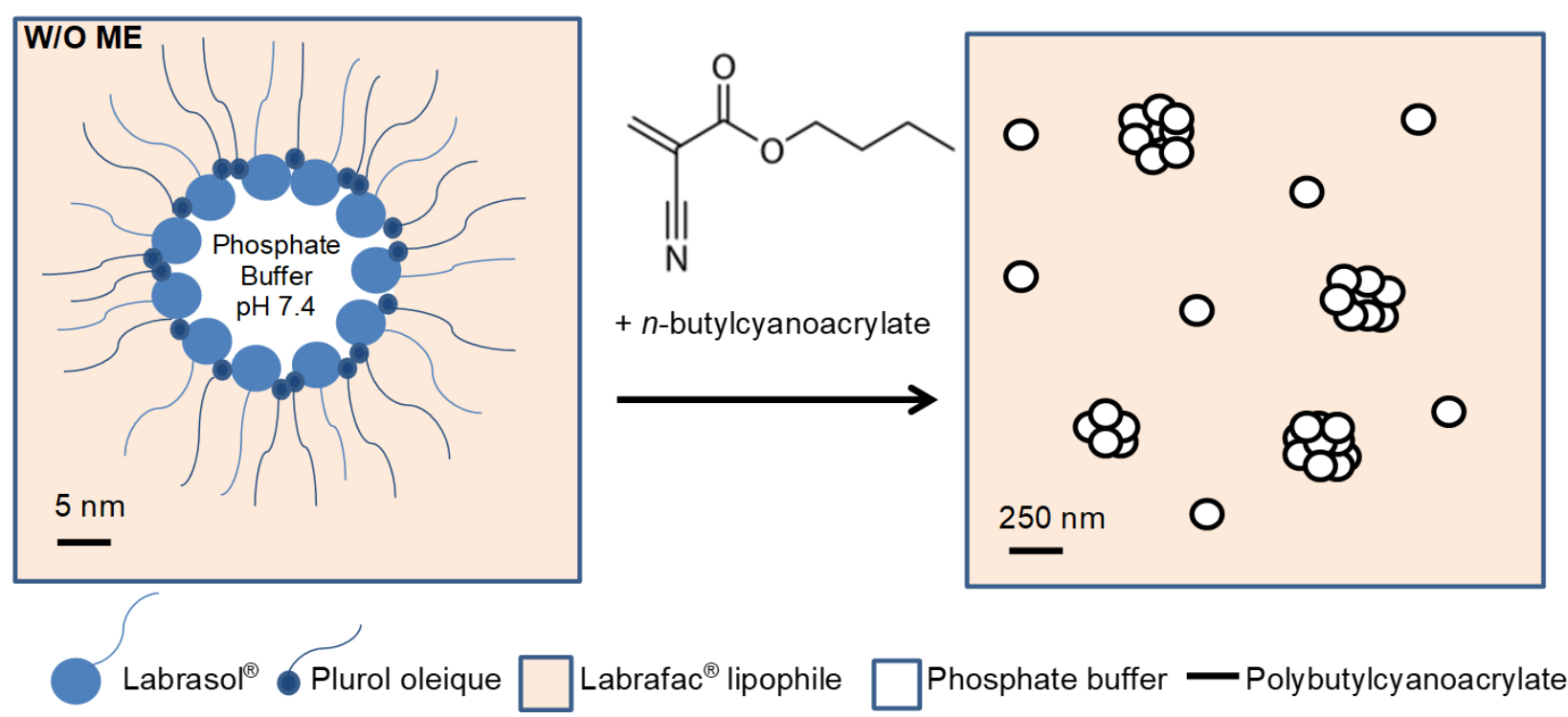




\section{Abstract}

Poly (n-butylcyanocrylate) nanocapsules were obtained by interfacial polymerization from a water-in-oil microemulsion. The latter was composed of pharmaceutically acceptable excipients such as Labrafac lipophile WL 1349 (oil), Plurol oleique CC497 (surfactant), Labrasol (co-surfactant) and was able to incorporate $20 \%$ aqueous phase. Pure n-butyl cyanoacrylate monomer (without any dilution in a halogenated solvent) was added to the microemulsion in order to obtain an oily suspension of biocompatible and biodegradable aqueous core nanocapsules. So, we provided a method of synthesis which allowed to increase by a large amount the quantity of nanocapsules synthesized at once. Finally, in order to provide a direct evidence of the presence of the aqueous core within the nanocapsules, a mass balance and nuclear magnetic resonance analysis were performed. The results showed the presence of water and Transmission Electronic Microscopy confirmed a hybrid structure of the final nanosystem, between a genuine nanocapsule and a matrix system: a nanosystem with multicavities filled with water.

KEYWORDS: aqueous core nanocapsules, water-in-oil microemulsion, n-butylcyanoacrylate, interfacial polymerization

\section{Introduction}

Discovered in the 40's by the chemists Hoar and Schulman[1], the microemulsion long remained a laboratory discovery before being used for the extraction of oil, after an oil shock. From the 90's, other applications emerged and particularly in the field of encapsulation. Infatuation ensued due to the ease with which the technique produced microemulsions. They could be inverse or direct and thus, allowed the encapsulation of either lipophilic or hydrophilic molecules. However, these systems were sensitive to thermodynamic modifications and therefore, released their encapsulated content very quickly. In addition, they were often composed of a high percentage of surfactant so compatibility problems arised for pharmaceutical uses.
The microemulsion could be used as a template and the aqueous nanodomains formed in a water-in-oil microemulsion could form the embryo of reservoirs in the nanocapsule structure.

Indeed, only a few nanoencapsulation processes provide aqueous core nanosystems such as liposomes [2, 3], polymersomes [4, 5], nanocapsules prepared by a double emulsionevaporation process [6] or by interfacial polymerization [7]. In the latter case, alkylcyanoacrylates are the most used monomers able to polymerize in presence of hydroxide anions provided by the dissociation of water molecules. In the late 70's, alkylcyanoacrylates were used to obtain nanospheres encapsulating hydrophilic molecules such as insulin [8]. Fifteen years later, Gasco and Trotta [9] prepared aqueous core- nanocapsules by interfacial polymerization of methylcyanoacrylate in water-in-oil (w/o) microemulsion. It is only at the beginning of the 2000s, with the work of Watnasirichaikul, et al, that the process was really studied [7] and evaluated for encapsulation of insulin $[6,9,10,11,12]$, small peptides [14], nucleosides [15, 16] or even proteins $[17,18]$ nanoencapsulation. However, among the alkylcyanoacrylates, only long chain alkylcyanoacrylates [19], like nbutylcyanoacrylate or octylcyanoacrylate, present the advantage of being biodegradable and biocompatible and therefore are FDAapproved for human use. Nevertheless, the solubilization of the monomer in halogenated organic solvents impairs the potential use of this approach.

As shown for nanocapsules prepared by interfacial polymerization of nbutylcyanoacrylate in reverse mini-emulsion $[20,21]$, the monomer could be directly added without prior dilution in chloroform but the dispersion required high shear energy to decrease droplet size. This can also be done in microemulsions using surfactants, and leads to nanocapsules using only "Generally Recognised As Safe" (GRAS) or more pharmaceutically acceptable excipients, without using any toxic organic solvents [22]. Last but not least, the microemulsion-based process should allow the formation of aqueous core-nanocapsules, i.e. with water reservoirs 
that would ensure the preservation of fragile hydrophilic molecules.

Therefore, we propose a suspension of fully biocompatible aqueous core-nanocapsules, made from a microemulsion composed of pharmaceutically acceptable excipients. In addition, by doubling the percentage of aqueous phase constituting the water-in-oil type microemulsion of reported studies in the literature, the final suspension contains a higher concentration of nanocapsules. Finally, while previous studies focused only on the encapsulation of hydrophilic drugs, the current paper provides direct evidence of the presence of an aqueous core within the nanocapsules obtained by interfacial polymerization. For this purpose, the obtained nanoparticles were observed by transmission electron microscopy in order to confirm the core-shell structure of the nanocapsules and by nuclear magnetic resonance to show the presence of water in the core.

\section{Experimental}

\section{Materials}

Labrafac lipophile WL1349 (caprylic/capric triglycerides) was purchased from Gattefossé (Saint-Priest, France). Labrasol (PEG8 caprylic/capric glycerides) and Plurol oleique CC497 (polyglyceryl-3-dioleate) were kindly provided by Gattefossé (Saint-Priest, France). The n-butylcyanoacrylate monomer (BCA) was kindly supplied by Henkel (Dublin, Ireland). Deuterium oxide (D2O) was purchased from Sigma-Aldrich (Saint-Quentin-Fallavier, France). Bovine serum albumin (BSA) ( $\geq 98 \%$ ) was provided by Sigma-Aldrich (SaintQuentin-Fallavier, France). All other chemical reagents were obtained from Sigma-Aldrich (Saint-Quentin-Fallavier, France) and Prolabo (Fontenay-sous-Bois, France) and were of analytical grade. Ultra-pure water was obtained from a Milli-Q® System (Millipore, Paris, France).

\section{Pseudo-ternary phase diagram preparation}

Pseudo-ternary phase diagram of oil (Labrafac lipophile WL 1349), surfactant (Plurol oleique CC497), co-surfactant (Labrasol) and water was built, based on the results obtained by
Chen, et al [23]. The surfactant and cosurfactant in a ratio $1 / 1(\mathrm{w} / \mathrm{w})$ and the oil were weighted in a screw-cap vial and vortexed. Water was then added and the vial was vortexed again. Vials were let overnight at room temperature to allow the content to equilibrate. Visual observation was used to identify the formation of microemulsions (limpid or translucid) or coarse emulsions (turbid).

\section{Conductivity}

Microemulsions were characterized with regard to conductivity at room temperature, using a conductimeter C561 (Consort, Turnhout, Belgium) coupled to a micro-volume electrode SK24T (Consort, Turnhout, Belgium). Samples for conductivity measurement were prepared using $10 \mathrm{mM}$ sodium chloride solution as the aqueous phase. For this $\mathrm{NaCl}$ concentration, the global thermodynamic equilibrium is maintained. The measurements were carried out in triplicate.

\section{Nanocapsule preparation}

The poly(n-butylcyanoacrylate) (PBCA) nanocapsules were prepared by interfacial anionic polymerization using a procedure adapted from Watnasirichaikul, et al [7]. Briefly, $1.75 \mathrm{~g}$ of Labrafac lipophile WL 1349, $1.125 \mathrm{~g}$ of Plurol oleique CC 497 and $1.125 \mathrm{~g}$ of Labrasol were mixed by magnetic stirring at room temperature for 15 minutes. Then, $1 \mathrm{~g}$ of phosphate buffer saline (PBS, pH 7.4) was added to this pre-microemulsion. The microemulsion was obtained within 15 seconds but the agitation was maintained during 15 minutes. At final, the microemulsion was composed by oil (35\%), surfactant/cosurfactant (w/w, 1/1) (45\%) and aqueous phase (20\%). Then, about $400 \mathrm{mg}$ of $\mathrm{n}$ butylcyanoacrylate monomer were drop-wise added to the microemulsion under magnetic stirring. The system was left for at least 12 hours at room temperature for polymerization. An oily suspension of aqueous-corenanocapsules was obtained and used per se or collected in water after ultracentrifugation. To obtain an aqueous suspension of nanocapsules, the system was centrifuged at 204,000 g, 1 hour, $20^{\circ} \mathrm{C}$ in an Optima TLX ultracentrifuge (Beckman Coulter, California, USA) with a TLA100.4 rotor. The oily supernatant was eliminated and PBCA nanocapsules were recovered and dispersed by soft sonication, 580 
W, during 20 secondes ( Fisherbrand type $\mathrm{P} 60 \mathrm{H}$, Singen, Germany) in a phosphate buffer saline ( $\mathrm{pH} 7.4)$, that does not destroy the particles as shown the TEM images.

For preparation of BSA-loaded nanocapsules, the protein was dissolved in PBS at a concentration of $5 \mathrm{mg} / \mathrm{mL}$. This aqueous phase was added to the oil and surfactants mixture and nanocapsules were then obtained according to the method described above.

\section{BSA loading and in vitro release}

For BSA loading assessment, $500 \mathrm{mg}$ of oily suspension of BSA-loaded nanocapsules were weighted into a centrifuge tube and were centrifuged at $204,000 \mathrm{~g}, 1$ hour, $20^{\circ} \mathrm{C}$ in an Optima TLX ultracentrifuge (Beckman Coulter, Fullerton, California, USA) with a TLA100.4 rotor. Tubes were then inverted and left for 30 minutes to remove the oily supernatant and recover the nanocapsule pellet. The nanocapsule pellet was rinsed with $1 \mathrm{~mL}$ of ethanol and was left for 30 minutes to evaporate. $100 \mu \mathrm{L}$ of acetone was added in order to solubilize nanocapsules during 2 hours. $900 \mu \mathrm{L}$ of PBS was then added and the tube was vortexed to extract the BSA. Finally, the tube was centrifuged at $204.000 \mathrm{~g}, 5 \mathrm{~min} ., 20^{\circ} \mathrm{C}$ and BSA concentration in supernatant was detected using a BCA protein assay kit (Pierce Biotechnology, Rockford, USA). For in vitro release, $1 \mathrm{~g}$ of oily suspension of BSA-loaded nanocapsules was weighted into a centrifuge tube and was centrifuged at 204,000 g, 1 hour, $20^{\circ} \mathrm{C}$ in an Optima TLX ultracentrifuge with a TLA100.4 rotor. Tubes were then inverted and left for 30 minutes to remove the oily supernatant and recover the nanocapsule pellet. The nanocapsule pellet was rinced twice by 1 $\mathrm{mL}$ of ethanol and was left for 30 minutes to evaporate. The nanocapsule pellet was resuspended in $2 \mathrm{~mL}$ PBS and placed in a shaker bath SW23 (Julabo GmbH, Seelbach, Germany) heated at $37^{\circ} \mathrm{C}$ and a shaken frequency set at 100 . The tubes were centrifuged at $87,000 \mathrm{~g}, 5 \mathrm{~min} ., 20^{\circ} \mathrm{C}$, at predetermined time intervals and $150 \mu \mathrm{L}$ of supernatant was collected. $150 \mu \mathrm{L}$ of fresh PBS was added and tubes were placed again into the shaker bath. Free BSA in the supernatant of each sample was then detected using the BCA protein assay kit.

\section{Physical stability}

The stability of the microemulsion and the oily suspension of nanocapsules obtained by butylcyanoacrylate polymerization was studied with the static multiple light scattering (S-MLS) technique (Turbiscan Tower, Formulaction, Toulouse, France). The S-MLS technique is the most suited optical method to characterize concentrated liquid dispersions in their native state. So, droplets (emulsion) or nanoparticles (suspension) could be characterized without dilution. $15 \mathrm{~mL}$ samples freshly prepared were placed in $30 \mathrm{~mL}$ vials. Turbiscan analyses were carried out over a period of 3 days, with one scan every 20 minutes ( 200 scans) at $25^{\circ} \mathrm{C}$. The sample was scanned from the bottom to the top by a near infrared light source $(\square=880 \mathrm{~nm})$ and two synchronous sensors collect the transmitted (T) and backscattered (BS) light signals. Finally, the apparatus translated he raw data of $\mathrm{T}$ and BS signals into Turbiscan Stability Index (TSI) which indicate the sample stability as a function of time. The TSI at time $t$ is calculated by summing-up all temporal and spatial variations:

$T S I(\mathrm{t})=\frac{1}{N_{h}} \sum_{t_{i}} \sum_{z_{i}}\left|B S T\left(t_{i}, z_{i}\right)-B S T\left(t_{i-1}, z_{i}\right)\right|$

with Nh the number of height positions in the selected zone of the scan, BST the considered signal (BS if $\mathrm{T}<0.2 \%, \mathrm{~T}$ otherwise). Consequently, the higher the TSI, the less stable the sample.

In addition, the mean size of droplets in emulsion or nanoparticles in suspension was determined with S-MLS. The mean diameter is deduced from $\mathrm{T}$ mean value thanks to the BeerLambert law [24]:

$\mathrm{T}=\mathrm{T}_{0} \exp \left(\frac{-2 \mathrm{r}_{\mathrm{i}}}{1}\right)=\mathrm{T}_{0} \exp \left(\frac{-3 \mathrm{r}_{\mathrm{i}} \phi \mathrm{Q}_{\mathrm{e}}(\mathrm{d})}{\mathrm{d}}\right)$

With T0 the transmitted signal without droplets or nanoparticles, 1 the mean free path representing the mean distance travelled by a photon between two particles, ri the radius of the cylindrical tube, $d$ the mean diameter, $\phi$ the volume fraction and $\mathrm{Qe}$ the extinction efficiency that can be calculated thanks to the Mie theory.

In the case of concentrated dispersions, the backscattered signal BS is used to evaluate the 
mean diameter thanks to the following equation [25]:

$\mathrm{BS}=\alpha \sqrt{\frac{1}{{ }^{*}}}+\beta=\alpha \sqrt{\frac{3 \phi(1-\mathrm{g}(\mathrm{d})) \mathrm{Q}_{\mathrm{e}}(\mathrm{d})}{2 \mathrm{~d}}}+\beta$

The transport mean free path $1^{*}=1 /[1-\mathrm{g}(\mathrm{d})]$ depends on $g$ the asymmetry factor quantifying the anisotropy of the light scattered by the particles and can be also calculated with the Mie theory. $\alpha$ and $\beta$ are calibration constant.

In a second step, size kinetic was followed on $15 \mathrm{~mL}$ microemulsion samples. Every 15 minutes, predetermined amount of nbutylcyanoacrylate monomer, until $1200 \mathrm{mg}$, was added under agitation and Turbiscan analyses were carried out over a period of 5 minutes, with one scan every 1 minute ( 5 scans) at $25^{\circ} \mathrm{C}$. Nanoparticle diameter was determined from each Turbiscan analysis following the procedure described above.

\section{Transmission Electron Microscopy}

Water suspension nanocapsule morphology was visualized by transmission electron microscopy (TEM) using a JEOL JEM-1400 transmission electron microscope equipped with a camera ORIUS SC1000 11MP. A diluted nanocapsules suspension at a concentration around $1 \mathrm{mg} / \mathrm{mL}$ was dropped off on Formvarfilmed nickel grid and left to dry overnight at room temperature. The observation deck was tilted to $45^{\circ}$ in order to obtain 3D-like pictures.

\section{Nuclear Magnetic Resonance (NMR)}

Nanocapsule NMR spectra were acquired on a Bruker $500 \mathrm{MHz}$ DRX spectrometer equipped with $5 \mathrm{~mm}$-broadband inverse probes (Bruker Biospin, Wissembourg, France).

Different quantities of oily nanocapsule suspension $(0.5,1$ and $1.5 \mathrm{~g})$ were centrifuged at $204,000 \mathrm{~g}, 1$ hour, $20^{\circ} \mathrm{C}$ in an Optima TLX ultracentrifuge with a TLA100.4 rotor. Tubes were then inverted and left for 30 minutes to remove the oily supernatant and to recover the nanoparticle pellet. These nanocapsule pellets were suspended in $1.5 \mathrm{~mL}$ of $\mathrm{D} 2 \mathrm{O}$ before observation.

\section{Mass balance analysis}

$500 \mathrm{mg}$ of oily suspension of nanocapsules were weighted into a tared centrifuge tube and were centrifuged at $204,000 \mathrm{~g}, 1$ hour, $20^{\circ} \mathrm{C}$ in an Optima TLX ultracentrifuge (Beckman Coulter, Fullerton, California, USA) with a TLA100.4 rotor. Tube was then inverted on another tared tube and left for 30 minutes to recover the oily supernatant. Then, tubes with supernatant or with pellet nanocapsules have been weighted. Samples were carried out in triplicate and compared to the theoretical weights.

\section{Thickness evaluation of the nanocapsule polymeric shell}

The polymeric volume per nanocapsule, $\mathrm{Vpn}$, is the difference between the volume of the nanocapsule and the volume of the nanodroplet and can be defined as follows:

$\mathrm{Vpn}=\frac{4}{3} \pi(R+r)^{3}-\frac{4}{3} \pi(R)^{3}$

where $\mathrm{R}$ is the nanodroplet radius and $\mathrm{r}$ the thickness of the polymeric shell.

So, the theoretical thickness of the nanocapsule polymeric shell $\mathrm{r}$ can be calculated from the following equation:

$r=\sqrt[3]{R^{3}+\frac{3 \cdot V p n}{4 . \pi}}-\mathrm{R}$

However and according to the difference in volumetric mass between the polymer and the water, this parameter has to be included. The equation is then:

$r=R \cdot\left(\sqrt[3]{1+\frac{M p \cdot D w}{M w \cdot D p}}-1\right)$

where $\mathrm{Mp}$ and $\mathrm{Mw}$ are the mass of polymer and water, respectively, and $\mathrm{Dp}$ and $\mathrm{Dw}$ are the volumetric mass of polymer and water, i. e. 989 $\mathrm{Kg} / \mathrm{m} 3$ and $1000 \mathrm{Kg} / \mathrm{m} 3$, respectively [26]. Finally, the expression of $r$ only depends on the nanodroplet radius $\mathrm{R}$ and the ratio $\mathrm{Mp} / \mathrm{Mw}$,

$r=R \cdot\left(\sqrt[3]{1+\frac{1.011 \cdot M p}{M w}}-1\right)$ 


\section{Results and discussion}

\section{Characterization of the w/o microemulsions}

\section{Pseudo-ternary phase diagram}

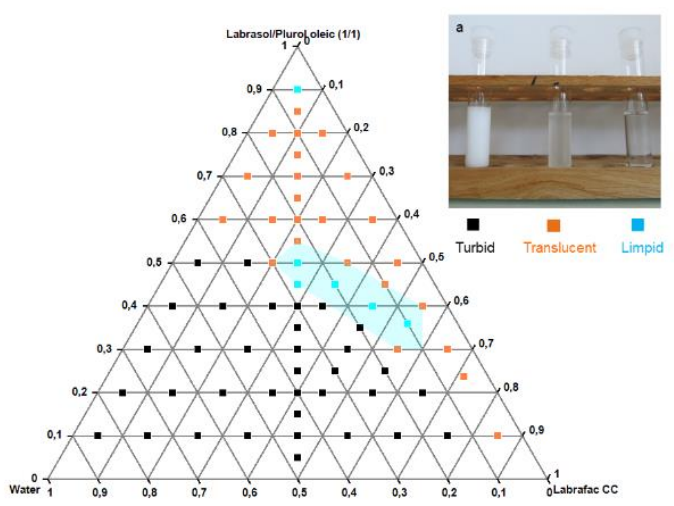

Fig.1: Pseudo-ternary phase diagram of the system (Labrafac CC/Labrasol/Plurol oleique CC 497/aqueous phase). Clear and limpid microemulsions were obtained in blue area. Physical aspects of ternary mix are illustrated in (a).

Fig. 1 represents a pseudo-ternary phase diagram of the mixture of Labrasol/ Plurol oleique CC497 (w/w, 1/1) as surfactants (top), Labrafac lipophile WL 1349 as oil (right), and water (left) at room temperature. A coarse emulsion was obtained in the turbid area whilst microemulsion was obtained in the rest of the diagram (clear limpid and translucent zone) (Fig 1-insert). The microemulsion transparency in this zone guaranteed stability and "nanodivision" of the aqueous phase. Indeed, the study of Sole et al [27] showed that the disappearance of the microemulsion clarity led to an increase of droplet size. Thus, the turbidity emergence was directly related to the increase of the microemulsion droplet size. Exploration of this pseudo-ternary phase diagram of a water-in-oil microemulsion enabled us to define a feasibility zone for the formation of nanocapsules containing up to $20 \%$ aqueous phase (Fig 1), twice the amount usually incorporated in these type of formulations [6,12-16]. To our knowledge, only the work of Graf et al $[10,11]$ reports the synthesis of poly(ethylcyanoacrylate) nanoparticles obtained from bicontinuous microemulsions containing up to $30 \%$ of aqueous phase. However, the nanoparticles obtained from these bicontinuous microemulsions encapsulated less insulin than those obtained from water-in-oil microemulsions, unless a significant increase in monomer was used [11].

\section{Microemulsion conductivity}

A conductivity study was performed to confirm the water-in-oil nature of the microemulsion. The conductivity was measured within the limpid zone as defined in Fig. 1 in which the water content ranged from 10 to $25 \%(\mathrm{w} / \mathrm{w})$ and in the coarse region in which water content ranged from $35 \%$ to $90 \%$ (w/w). As shown in Fig. 2, conductivity in the limpid area slightly increased with water content (Fig. 2 -insert) but remained low $(<15 \mu \mathrm{S} / \mathrm{cm})$, confirming that a water-in-oil microemulsion is obtained. Indeed, it is now accepted that the conductivity increases with the increase of aqueous phase within the microemulsion[28]. So, for low volume fractions of aqueous phase in the medium, nanodroplets were isolated in the continuous oily phase and the microemulsion conductivity remained low. According to the percolation theory, the conductivity increased exponentially from a threshold in the volume fraction of the dispersed phase[29]. From this point, a nanodoplet agglomeration formed an open structure that promoted a conductivity increase.

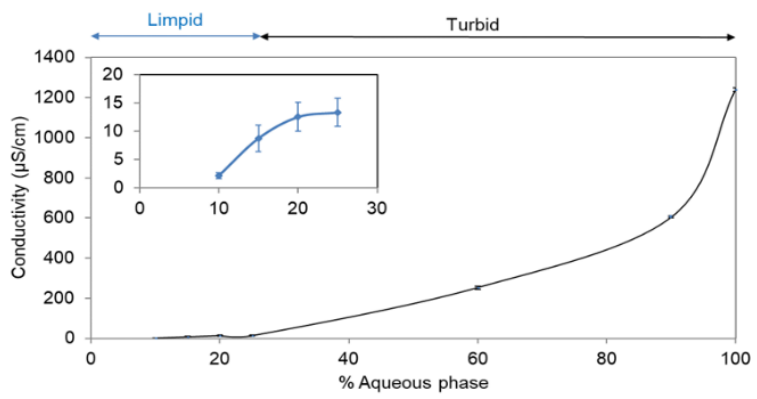

Fig. 2: Conductivity of microemulsion as a function of water concentration (\%). Values represent mean \pm standard deviation, $n=3$.

In the coarse region, conductivity measurements were characteristic of a direct emulsion. In the following, nanocapsules were made from a water-in-oil microemulsion composed of 35\% Labrafac lipophile WL1349, $45 \%$ of a mixture Labrasol / Plurol oleique CC497 in a ratio $1 / 1$ and 20\% PBS.

\section{Physical stability}

The Turbiscan tower allowed the follow-up of emulsion or suspension stability. It gives indications on their stability and anticipates 
sedimentation, coalescence or creaming phenomena well before they are visible to the naked eye.

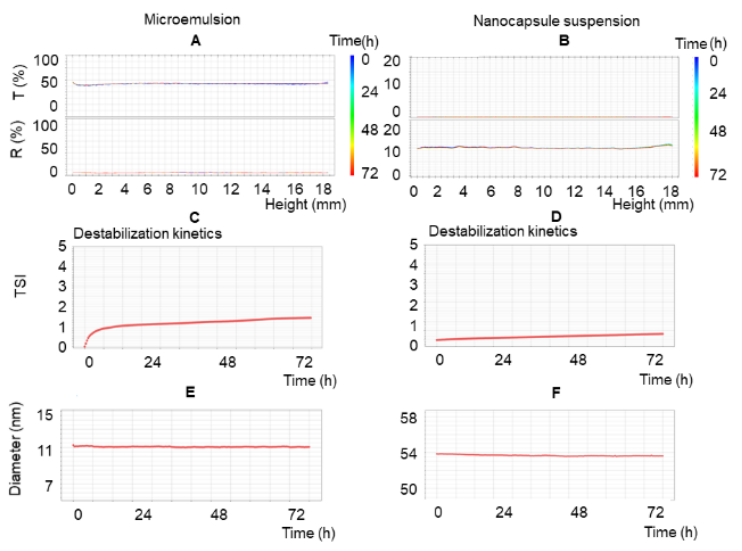

Fig. 3: Stability of microemulsion and polybutylcyanoacrylate nanoparticule suspension over a 3 days period.

Transmission and retrodiffusion curves of microemulsion (A) and nanocapsule suspension (B). TSI evolution for microemulsion (C) and nanocapsule suspension (D).

Size evolution of microemulsion (E) and nanocapsule suspension (F).

Fig. $3 a$ and $3 b$ showed the transmission and backscattering curves for the microemulsion and the suspension of nanocapsules, respectively, over a 3-day period of time. Microemulsion was analysed by the transmission curve which did not vary during the period of analysis. Nanocapsule suspension that became white opaque after polymerization of n-butylcyanoacrylate was analyzed in retrodiffusion since the transmission fell below $0.2 \%$. The linearity of the curves showed that no modification occurred for both the microemulsion and the nanocapsule suspension over a 3-day period. The stability was quantified via the Turbiscan stability index (TSI) which was below 5 for both the microemulsion and the nanocapsule suspension, threshold where destabilization occurs (Fig. 3c \&d).

In addition, no change in size over time was observed in either preparation. The nano-object diameter of the microemulsion was $10.5 \mathrm{~nm} \pm$ $0.5 \mathrm{~nm}$ (Fig. 3e). After polymerization, the average diameter of the nanocapsules was 50.8 $\mathrm{nm} \pm 1.0 \mathrm{~nm}$ (Fig. 3f).

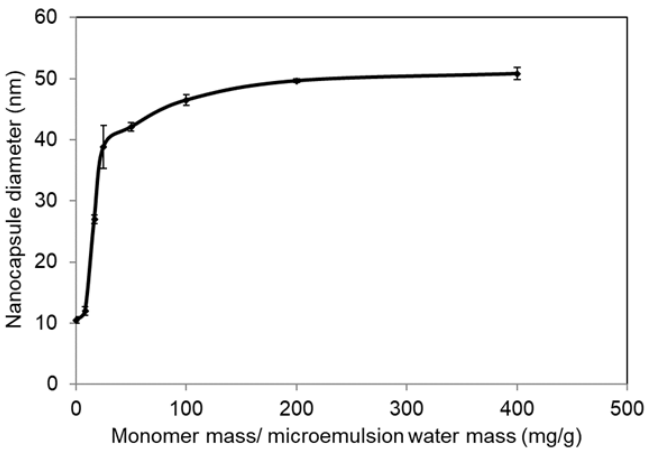

Fig.4: Nanocapsule diameter as function of monomer mass $(n=3)$

Mean nanocapsule diameter as a function of monomer quantity added to the preparation is shown in Fig. 4. A very rapid increase in the average diameter of the nanocapsules was observed as soon as the first milligrams of monomer were added. A plateau was then reached for a mean diameter value around 50 $\mathrm{nm}$ as soon as $10 \%$ of the maximum monomer mass was added.

\section{Nanocapsule characterizations}

The transmission electron microscopy pictures (Fig. 5) confirmed the presence of small structures in nanoparticles with diameter of ca $10 \mathrm{~nm}$ corresponding to the diameter of the nanodomains of aqueous phase in the microemulsion, measured by the Turbiscan tower (Fig. 3). However and as shown in Fig. 5, ca $200 \mathrm{~nm}$ multicavity particles were observed. Thus, it could be hypothesized that the formed nanocapsules were the result of an aggregation of individual $10 \mathrm{~nm}$-nanocapsules. Moreover, the $3 \mathrm{D}$-like picture obtained by tilting to $45^{\circ}$ the observation deck (Fig. 5b) confirmed that nanoparticles were formed of an agglomeration of individual nanocapsules (though $10 \mathrm{~nm}$ individual nanocapsules were still present). The agglomeration observed could result from an excess of monomer added to the reaction medium. Indeed, Musumeci, et al, [21] using nbutylcyanoacrylate at $7 \% \mathrm{w} / \mathrm{w}$ ratio in their nanoencapsulation process reported the formation of nanoparticles exhibiting multicavities, whereas Hillaireau, et al, [15, 16] using isobutylcyanoacrylate at $0.11 \% \mathrm{w} / \mathrm{w}$ ratio in their process obtained individualized nanocapsules. Similarly, the work of Krauel, et al, [18] suggested an aggregation of individual nanocapsules, called micelle-clusters to form 


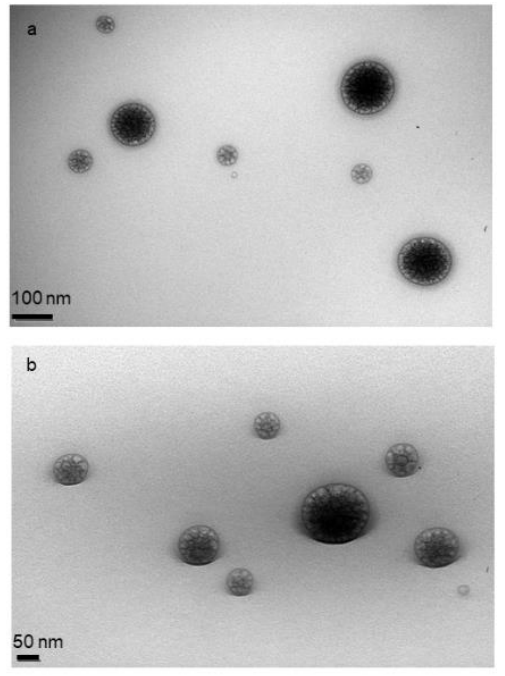

Fig.5: Transmission Electron Microscopy of polybutylcyanoacrylate nanocapsules after dispersion in water.

bigger nanoparticles having a size of $250 \mathrm{~nm}$, as observed in TEM (Fig. 5). The curve presenting the nanocapsule diameter evolution as a function of the added monomer mass would tend to confirm this result (Fig. 4), even if the mean diameter observed on this curve does not exceed $50 \mathrm{~nm}$. In fact, this could reflect a large disparity in size within the nanocapsules suspension. Despite the stirring of the reaction medium and the gradual addition of the monomer, it was likely that the instantaneous nature of the n-butylcyanoacrylate polymerization outweighed the diffusion of the monomer into the medium. This resulted in the coexistence of unpolymerized water nanodroplets, individualized nanocapsules and nanocapsule agglomeration.

Physico-chemical characterization of the synthetized nanocapsules indubitably showed that the core was constituted with water. To the best of our knowledge, all the reported studies in the literature show indirect proofs of a water core via the entrapment of hydrophilic molecules. Such indirect proofs were also given by the conclusive encapsulation of BSA (Fig. 6). Interestingly, despite its high molecular weight, the BSA did not destabilize the microemulsion, which was the first step in the formulation of nanocapsules. At concentration of $5 \mathrm{mg} / \mathrm{mL}$ in the aqueous phase, similar to the one used for the encapsulation of insulin[7, 10-12], protein[18] or peptide[14], an encapsulation efficiency close to $100 \%$ was

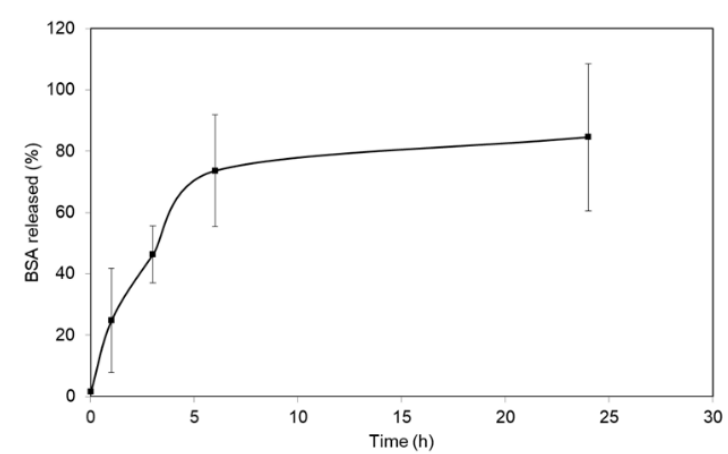

Fig. 6: Release of BSA in $\mathrm{pH} 7.4$ phosphate buffer from PBCA nanocapsules $(n=3)$

reached. The in vitro release study (Fig.6) showed a biphasic profile with a fast release period ( $50 \%$ of the BSA over the first 4 hours) and a slow release period, with mostly complete released at 24 hours, as generally observed in the literature for nanocapsules obtained by interfacial polymerization of water-in-oil microemulsion used as template [7, 14, 15]. Only the work of Musumeci, et al,[21] proposed a slower release of D-cycloserine from polybutylcyanoacrylate nanocapsules over more than a week. However, according to the authors, it is likely that part of the molecule was trapped in the nanoparticle polymeric shell.

In order to provide a first direct evidence of water in those nanoparticles, a mass balance analysis was performed (table 1) comparing the theoretical and observed masses of nanoparticle pellets (constituted of water and coating polymer) and the corresponding supernatant masses (containing oils and surfactants) after ultracentrifugation of the samples. No differences were observed between the theoretical and the actual weights showing that, in order to respect the mass balance, water had to be taken into account in the nanocapsule pellet mass.

\begin{tabular}{|c|c|c|c|c|}
\hline \multirow{2}{*}{} & \multicolumn{2}{|c|}{ Nanocapsule pellet } & \multicolumn{2}{c|}{ Supernatant } \\
\cline { 2 - 5 } & theoretical & observed & theoretical & observed \\
\hline Mass & & $134.22+/-$ & & $339.15+/-$ \\
(mg) & 129.65 & 3.76 & 370.35 & 8.89 \\
\hline & & $26.84+/-$ & & $67.83+/-$ \\
$\%$ & 25.93 & 0.75 & 74.07 & 1.78 \\
\hline
\end{tabular}

Tab. 1: Mass balance of polymerized microemulsion after centrifugation (theory and observed $(n=3)$ )

According to Turbiscan measurements of the diameter of nanocapsules after polymerization showing a mean diameter close to $50 \mathrm{~nm}$ (Fig. 3f) and as the microemulsion nanodroplets had 
a mean diameter close to $10 \mathrm{~nm}$ (Fig.3e), the polymeric shell should be about $20 \mathrm{~nm}$ thick. That was not consistent with the $0.6 \mathrm{~nm}$ thickness calculated from equation (7) for the mass of product used in the manufacturing (table 2). TEM (Fig. 5), confirms this thin shell and that polymerized nanocapsules were mainly present as agglomerates composed of 10 nm diameter capsules.

\begin{tabular}{|c|c|c|c|c|}
\hline $\begin{array}{c}\text { Water } \\
\text { mass } \\
\text { used }\end{array}$ & $\begin{array}{c}\text { Monomer } \\
\text { mass used }\end{array}$ & $\begin{array}{c}\text { Ratio } \\
\text { monomer } \\
\text { /water }\end{array}$ & $\begin{array}{c}\text { Nanodroplet } \\
\text { radius }\end{array}$ & $\begin{array}{c}\text { Thickness } \\
\text { evaluation }\end{array}$ \\
\hline $\mathrm{Me}$ & $\mathrm{Mp}$ & $\mathrm{Rmw}$ & $\mathrm{R}$ & $\mathrm{r}$ \\
\hline$(\mathrm{Kg})$ & $(\mathrm{Kg})$ & - & $(\mathrm{m})$ & $(\mathrm{m})$ \\
\hline $1.10^{-6}$ & $4.10^{-7}$ & 0.4 & $5.5 .10^{-9}$ & $6.59 .10^{-10}$ \\
\hline
\end{tabular}

Tab. 2: Thickness calculation of the nanocapsule shell

In order to provide a second direct evidence of the presence of water within the nanocapsules, increasing amounts of nanoparticles dispersed in the oily phase were ultracentrifuged.

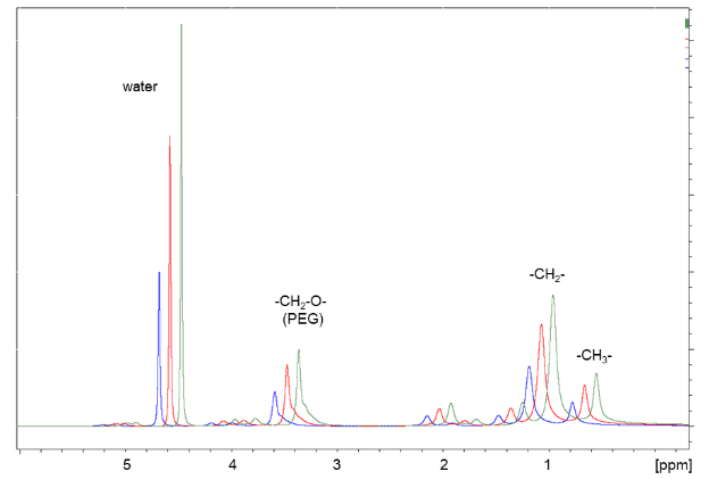

Fig. 7: NMR spectrum of $0.5 \mathrm{~g}$ (blue), $1.0 \mathrm{~g}$ (red) and $1.5 \mathrm{~g}$ (green) of nanoparticle pellet. For visualization purpose, the red and green spectra are shifted by 0.25 and $0.5 \mathrm{ppm}$ respectively.

Once the supernatant was removed, each nanoparticle pellet was resuspended in the same volume of deuterium oxide and analyzed by nuclear magnetic resonance. Fig. 7 presents the spectra of the three pellets of nanoparticles obtained after ultracentrifugation of $0.5 \mathrm{~g}, 1.0 \mathrm{~g}$ and $1.5 \mathrm{~g}$ nanoparticles in suspension. There was indeed a proportional increase for all the peak areas corresponding to the nanocapsule shell as well as the peak areas corresponding to water $(4.75 \mathrm{ppm})$ as the amount of nanoparticles in suspension increased, evidencing the presence of water within nanoparticles obtained by n-butylcyanoacrylate interfacial polymerization from a water-in-oil microemulsion, used as template. Of note, the polymerization of n-butylcyanoacrylate under these conditions was complete since no trace of monomer was found during the NMR analysis of the samples.

\section{Conclusions}

Nanocapsules containing an aqueous phase were obtained by the interfacial polymerization technique involving a water-in-oil microemulsion used as a template. The waterin-oil microemulsion was interesting as a template as it allowed dividing the aqueous phase into nanodomains without shear energy or heat. The monomer polymerization occurred around these aqueous nanodomains, allowing their entrapment. The presence of water inside the nanocapsules has been demonstrated, in particular by the NMR study. And, as showed by Transmission Electronic Microscopy, nanocapsules with nanodomains filled with water were obtained.

Now, hydrophilic molecules of interest should be encapsulated in these nanocapsules for sustained release and targeting purposes, even if much more formulation work has to be done in order to achieve such goal. A preliminary study, conducted with BSA and characterized in terms of encapsulation yield and release, showed the potentialities of such nanocapsules. Future studies may focus on improving and increasing the release kinetics of different proteins for which the primary and secondary structures are surely key parameters controlling their release from the final nano-object. Furthermore, it will be interesting to use this microemulsion template for other structuration phenomena such as natural polyelectrolyte associations, leading to more bioavailable and biodegradable nanoparticles for encapsulation and release of hydrophilic compounds.

\section{Acknowledgements}

We are grateful to $\mathrm{Mr}$ B. Siegler for NMR analysis, Mr R. Mallet for transmission electron microscopy and Mr P. Legout for BSA release kinetics. Internal University funds supported this research.

\section{Conflicts of Interest}

The authors declare no competing interests. 


\section{References}

1. Hoar TP, Schulman JH (1943) Transparent Water-in-Oil Dispersions: the Oleopathic Hydro-Micelle. Nature 152:102

2. Bordi F, Cametti C, Sennato S, Diociaiuti M (2006) Direct evidence of multicompartment aggregates in polyelectrolyte-charged liposome complexes. Biophysical Journal 91:1513-1520. https://doi.org/10.1529/biophysj.106.085142

3. McClements DJ (2015) Encapsulation, protection, and release of hydrophilic active components: Potential and limitations of colloidal delivery systems. Advances in Colloid and Interface Science 219:27-53. https://doi.org/10.1016/j.cis.2015.02.002

4. Levine DH, Ghoroghchian PP, Freudenberg J, et al (2008) Polymersomes: A new multifunctional tool for cancer diagnosis and therapy. Methods 46:25-32. https://doi.org/10.1016/j.ymeth.2008.05.006

5. Hu M, Shen Y, Zhang L, Qiu L (2016) Polymersomes via self-assembly of amphiphilic $\beta$ cyclodextrin-centered triarm star polymers for enhanced oral bioavailability of water-soluble chemotherapeutics. Biomacromolecules 17:1026-1039. https://doi.org/10.1021/acs.biomac.5b01676

6. Lakshmi, Singh S, Vijayakumar MR, Dewangan HK (2018) Lipid based aqueous core nanocapsules (ACNs) for encapsulating hydrophillic vinorelbine bitartrate: Preparation, optimization, characterization and in vitro safety assessment for intravenous administration. Curr Drug Deliv 15:1284-1293. https://doi.org/10.2174/1567201815666180716112457

7. Watnasirichaikul S, Davies NM, Rades T, Tucker IG (2000) Preparation of biodegradable insulin nanocapsules from biocompatible microemulsions. Pharm Res 17:684-689

8. Couvreur P, Roland M, Speiser P (1982) Submicroscopic biodegradable particles containing a biologically active substance and composition containing them. US patent 4329332

9. Gasco MR, Trotta M (1986) Nanoparticles from microemulsions. International Journal of Pharmaceutics 29:267-268. https://doi.org/10.1016/0378-5173(86)90125-0

10. Graf A, Jack KS, Whittaker AK, et al (2008) Protein delivery using nanoparticles based on microemulsions with different structure-types. European Journal of Pharmaceutical Sciences 33:434-444. https://doi.org/10.1016/j.ejps.2008.01.013

11. Graf A, Rades T, Hook SM (2009) Oral insulin delivery using nanoparticles based on microemulsions with different structure-types: Optimisation and in vivo evaluation. European Journal of Pharmaceutical Sciences 37:53-61. https://doi.org/10.1016/j.ejps.2008.12.017

12. Watnasirichaikul S, Rades T, Tucker IG, Davies NM (2002) Effects of formulation variables on characteristics of poly (ethylcyanoacrylate) nanocapsules prepared from w/o microemulsions. International Journal of Pharmaceutics 235:237-246. https://doi.org/10.1016/S0378-5173(02)00002-9

13. Graf A, Ablinger E, Peters S, et al (2008) Microemulsions containing lecithin and sugar-based surfactants: Nanoparticle templates for delivery of proteins and peptides. International Journal of Pharmaceutics 350:351-360. https://doi.org/10.1016/j.ijpharm.2007.08.053

14. Liang M, Davies NM, Toth I (2008) Increasing entrapment of peptides within poly(alkyl cyanoacrylate) nanoparticles prepared from water-in-oil microemulsions by copolymerization. International Journal of Pharmaceutics 362:141-146. https://doi.org/10.1016/j.ijpharm.2008.06.005 
15. Hillaireau H, Le Doan T, Besnard M, et al (2006) Encapsulation of antiviral nucleotide analogues azidothymidine-triphosphate and cidofovir in poly(iso-butylcyanoacrylate) nanocapsules. International Journal of Pharmaceutics 324:37-42. https://doi.org/10.1016/j.ijpharm.2006.07.006

16. Hillaireau H, Le Doan T, Chacun H, et al (2007) Encapsulation of mono- and oligonucleotides into aqueous-core nanocapsules in presence of various water-soluble polymers. International Journal of Pharmaceutics 331:148-152. https://doi.org/10.1016/j.ijpharm.2006.10.031

17. Pitaksuteepong T, Davies NM, Tucker IG, Rades T (2002) Factors influencing the entrapment of hydrophilic compounds in nanocapsules prepared by interfacial polymerisation of water-in-oil microemulsions. European Journal of Pharmaceutics and Biopharmaceutics 53:335342. https://doi.org/10.1016/S0939-6411(01)00245-4

18. Krauel K, Davies NM, Hook S, Rades T (2005) Using different structure types of microemulsions for the preparation of poly(alkylcyanoacrylate) nanoparticles by interfacial polymerization. Journal of Controlled Release 106:76-87. https://doi.org/10.1016/j.jconre1.2005.04.013

19. Korde JM, Kandasubramanian B (2018) Biocompatible alkyl cyanoacrylates and their derivatives as bio-adhesives. Biomaterials Science 6:1691-1711. https://doi.org/10.1039/C8BM00312B

20. Musyanovych A, Landfester K (2008) Synthesis of poly(butylcyanoacrylate) nanocapsules by interfacial polymerizationin miniemulsions for the delivery of DNA molecules. In: Auernhammer GK, Butt H-J, Vollmer D (eds) Surface and Interfacial Forces - From Fundamentals to Applications. Springer Berlin Heidelberg, pp 120-127

21. Musumeci T, Ventura CA, Carbone C, et al (2011) Effects of external phase on Dcycloserine loaded W/O nanocapsules prepared by the interfacial polymerization method. European Journal of Medicinal Chemistry 46:2828-2834. https://doi.org/10.1016/j.ejmech.2011.04.003

22. regulatory data sheet - pharmaceutical market: Labrasol (code : 3074), Plurol oleique CC497 (code : 3212) and Labrafac lipophile WL1349 (code : 3139), Gattefosse SAS, Saint Priest, France

23. Cheng M-B, Wang J-C, Li Y-H, et al (2008) Characterization of water-in-oil microemulsion for oral delivery of earthworm fibrinolytic enzyme. Journal of Controlled Release 129:41-48. https://doi.org/10.1016/j.jconrel.2008.03.018

24. Mazloumi M, Johnston LJ, Jakubek ZJ (2018) Dispersion, stability and size measurements for cellulose nanocrystals by static multiple light scattering. Cellulose 25:5751-5768. https://doi.org/10.1007/s10570-018-1961-6

25. Mengual O (1999) TURBISCAN MA 2000: multiple light scattering measurement for concentrated emulsion and suspension instability analysis. Talanta 50:445-456. https://doi.org/10.1016/S0039-9140(99)00129-0

26. O’Neil NJ (2006) The Merck Index : an encyclopedia of chemicals, drugs \& biologicals, 14th edition New Jersey

27. Solè I, Solans C, Maestro A, et al (2012) Study of nano-emulsion formation by dilution of microemulsions. Journal of Colloid and Interface Science 376:133-139. https://doi.org/10.1016/j.jcis.2012.02.063 
28. Fanun M (2008) Phase behavior, transport, diffusion and structural parameters of nonionic surfactants microemulsions. Journal of Molecular Liquids 139:14-22. https://doi.org/10.1016/j.molliq.2007.10.005

29. Moulik SP, Ray S (1994) Thermodynamics of clustering of droplets in water/AOT/heptane microemulsion. Pure and
https://doi.org/10.1351/pac199466030521

Applied Chemistry 66:521-525. 